CARTA AL EDITOR

\title{
Ventilador mecánico: ¿recurso compartible?
}

\section{Mechanical ventilator: shared resource?}

\section{José L. Sandoval-Gutiérrez*}

Subdirección de Servicios Auxiliares de Diagnóstico y Paramédicos, Instituto Nacional de Enfermedades Respiratorias Ismael Cosío Villegas, Ciudad de México, México

Vázquez de Anda et al. ${ }^{1}$ publican un artículo que emprende el posible viaje de contestar las connotaciones bioéticas de una justicia distributiva, sobre la disponibilidad de un ventilador mecánico para al menos dos pacientes en una situación de extrema necesidad de este, en estado de emergencia o pandemia como es el actual caso de la enfermedad por coronavirus 2019.

Hacen referencia a la patente del grupo de la Universidad de Yale denominado multiplexors que fue autorizado para caso de emergencia por la Food and Drug Administration de EE.UU., pero no de forma sistemática. Los autores concluyen: "Con esta medida se atiende al mayor número de personas con la misma probabilidad de salvar a un mayor número de personas".
Desgraciadamente, esta sentencia aún cae en el terreno de la posibilidad, ya que nadie lo ha demostrado y será difícil que un comité de bioética institucional apruebe un estudio prospectivo de ventilación única frente a ventilación dual en situación de no pandemia, por lo que la conclusión se podría parafrasear de la siguiente manera:

"Con esta medida se desatiende al mayor número de personas con la misma probabilidad de no salvar a un mayor número de personas".

\section{Bibliografía}

1. Vázquez de Anda GF, Ruíz de Chávez M, Pérez Castañeda Al, Vázquez Moreno P, Dávila Fernández JC, Delaye Aguilar MG. El ventilador mecánico como recurso divisible ante la pandemia del COVID19. Gac Med Mex. 2020;156:306-10. CC BY-NC-ND (http://creativecommons.org/licenses/by-nc-nd/4.0/). 